\title{
Adenomatoid odontogenic tumor with impacted mandibular canine: a case report
}

\author{
Sunil S. Mishra ${ }^{1}$, Shirish S. Degwekar ${ }^{2}$, Mukta B. Motwani ${ }^{3}$, Ruchi M. Anand ${ }^{4}$ \\ ${ }^{1}$ B.D.S.Academic Post: Post Graduate Student (Oral Medicine and Radiology), Department of Oral Medicine \& Radiology, \\ Sharad Pawar Dental College \& Hospital, Sawangi (Meghe), Wardha, Maharashtra State, India. \\ ${ }^{2}$ B.D.S., M.D.S. (Oral Medicine and Radiology), Professor and Guide, Department of Oral Medicine \& Radiology, Sharad Pawar \\ Dental College \& Hospital, Sawangi (Meghe), Wardha, Maharashtra State, India. \\ ${ }^{3}$ B.D.S., M.D.S. (Oral Medicine and Radiology), Professor Department of Oral Medicine \& Radiology V.S.P.M. Dental College \\ \& Hospital, Hinghna, Nagpur, Maharashtra State, India. \\ ${ }^{4}$ B.D.S., P. G. Diploma student (Oral Medicine and Radiology), Department of Oral Medicine \& Radiology, Sharad Pawar Den- \\ tal College \& Hospital, Sawangi (Meghe), Wardha, Maharashtra State, India.
}

\author{
Mishra SS, Degwekar SS, Motwani MB, Anand RM. Adenomatoid odon- \\ togenic tumor with impacted mandibular canine: a case report. J Clin Exp \\ Dent. 2011;3(Suppl1):e373-6 \\ http://www.medicinaoral.com/odo/volumenes/v3iSuppl1/jcedv3iSu- \\ ppl1p373.pdf
}

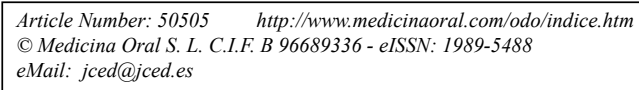

\begin{abstract}
The Adenomatoid Odontogenic Tumor (AOT) is a rare, slow growing, benign, odontogenic epithelial tumor with characteristic clinical and histological features; which usually arise in the second or third decade. It is a tumor composed of odontogenic epithelium in a variety of histoarchitectural patterns which are embedded in a mature connective tissue stroma. It is mostly encountered in young patients with a greater predilection for females. Maxilla is the predilection site of occurrence, most commonly associated with an unerupted maxillary canine. It presents as a symptom-free lesion and is frequently discovered during routine radiographic examination. This case report describes an unusual case of 20 year old male with only a one month history of tumor in the anterior mandible. The tumor was a well circumscribed intraosseous lesion with an embedded tooth. Histological evidence of calcification was present. The present case lends support to the categorization of AOT as a mixed odontogenic tumor.
\end{abstract}

Key words: Adenomatoid Odontogenic Tumor, impacted tooth, mandible, cuspid. 


\section{Introduction}

The Adenomatoid Odontogenic Tumor (AOT) is a rare, slow growing, benign, odontogenic epithelial tumor with characteristic clinical and histological features; which usually arise in the second or third decade. (1, 2) Most AOT occur intraosseously. They surround the crowns and are attached to the necks of unerupted teeth in a true follicular relationship. Some however have no association with unerupted teeth and a few arise on the gingiva in an extraosseous location. (3) The tumor affects females more than males in almost a two to one ratio. The maxilla is involved nearly twice as frequently as the mandible of which the canine region is the most commonly involved region. $(2,4)$ We herein report a case of AOT arising from mandibular canine region (follicular variant).

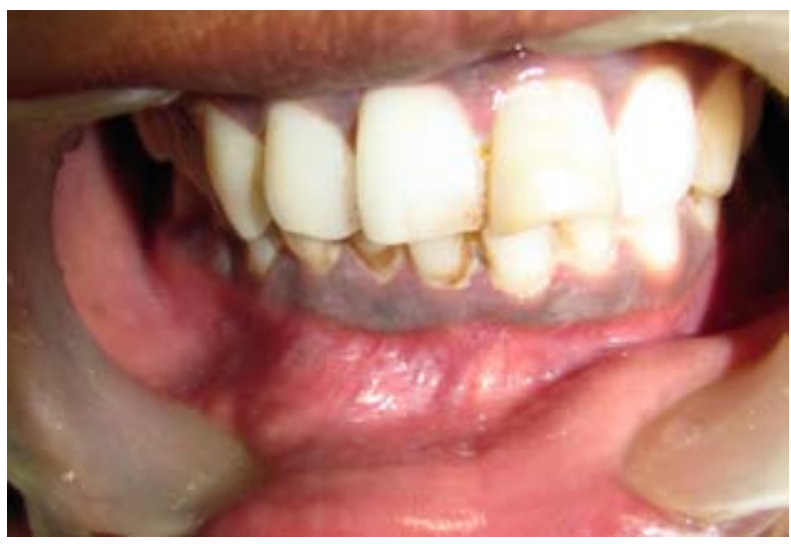

Fig. 1. A. Intraoral view showing a hyperemic diffuse area with the labial vestibular region along with clinically missing right mandibular permanent canine.

\section{Case Report}

A 20 year old male patient presented in the Department of Oral Medicine and Radiology, Sharad Pawar Dental College, with a one month history of swelling in the lateral-canine area of right mandible. Extraoral examination disclosed a diffuse firm swelling in the anterior mandible with no pain. When examined intraorally (Figure 1A) it was seen that right mandibular canine was clinically missing and was occupied by an overretained deciduous canine. There was also a hyperemic swelling in the lower labial vestibule with the right canine area. On radiological examination (intraoral, panoramic) a radiolucent lesion with a regular border was seen in the area ranging between the right mandibular premolar and left canine (Figure 1B). The lesion was unilocular and was involving the impacted right mandibular canine in the apex of mandibular central and lateral incisors. There was thinning of the inferior cortex at the symphyseal region. There was irregular root resorption seen with right and left central and lateral incisors, right premolars and left canine. A 3-dimensional x-ray and Computed Tomography scan revealed buccal cortical plate expansion along with thinning of lingual cortical plates (Figure 2 A, B).

Preoperative intentional root canal treatment was done with the mandibular anterior teeth and right premolar for better prognosis. Under the clinical diagnosis of dentigerous cyst, enucleation of the lesion with the extraction of the impacted canine was done. The specimen was sent for histological examination which showed the presence of rosettes with eosinophilic material filling the ductal lumen along with areas of calcification which suggested a diagnosis of AOT (Figure 3). A 6-month follow-up has been completed with no signs of recurrence.

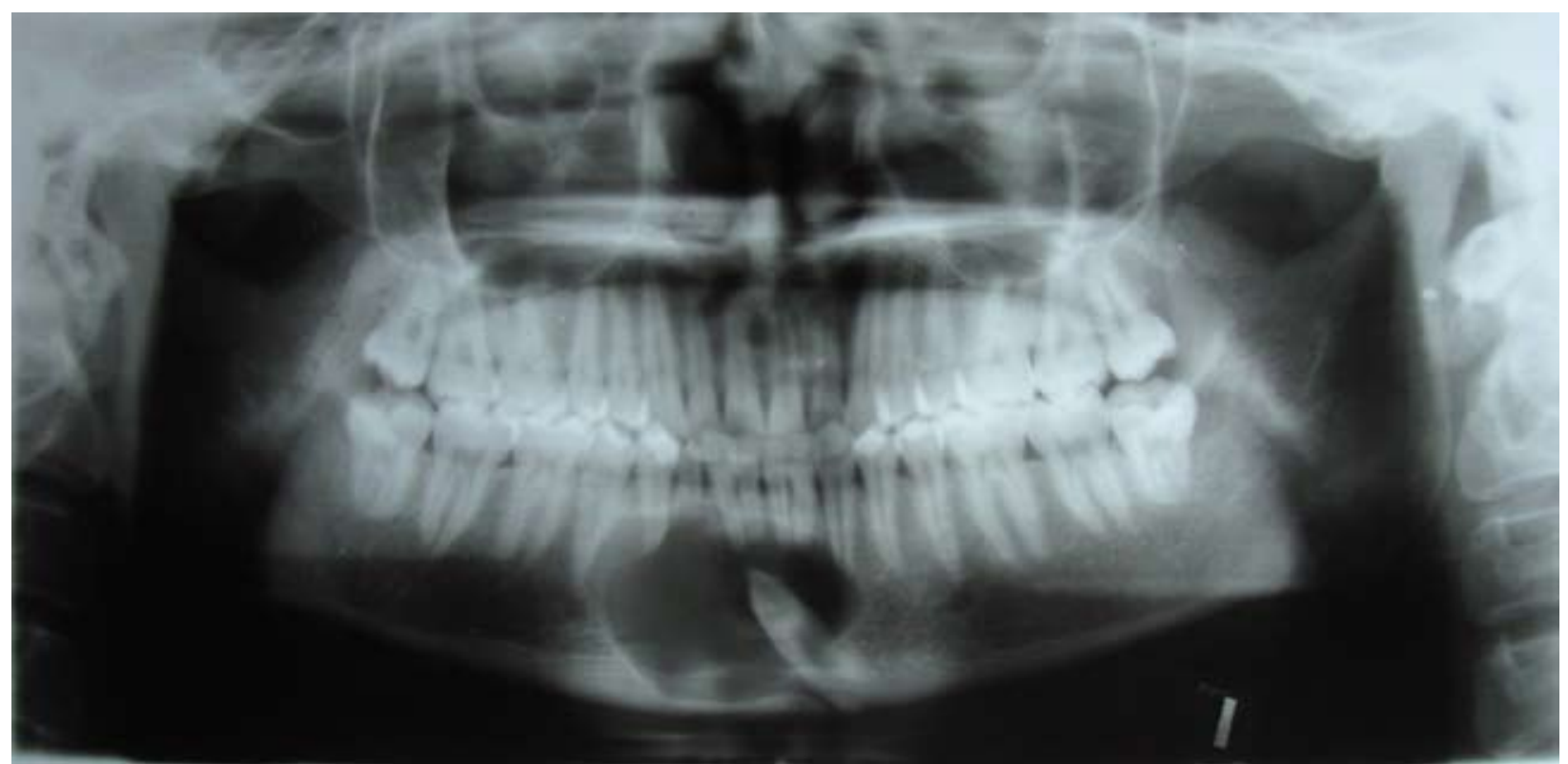

Fig. 1. B. Panoramic radiograph showing a well defined radiolucent lesion in the anterior mandibular region involving the impacted right mandibular canine. 


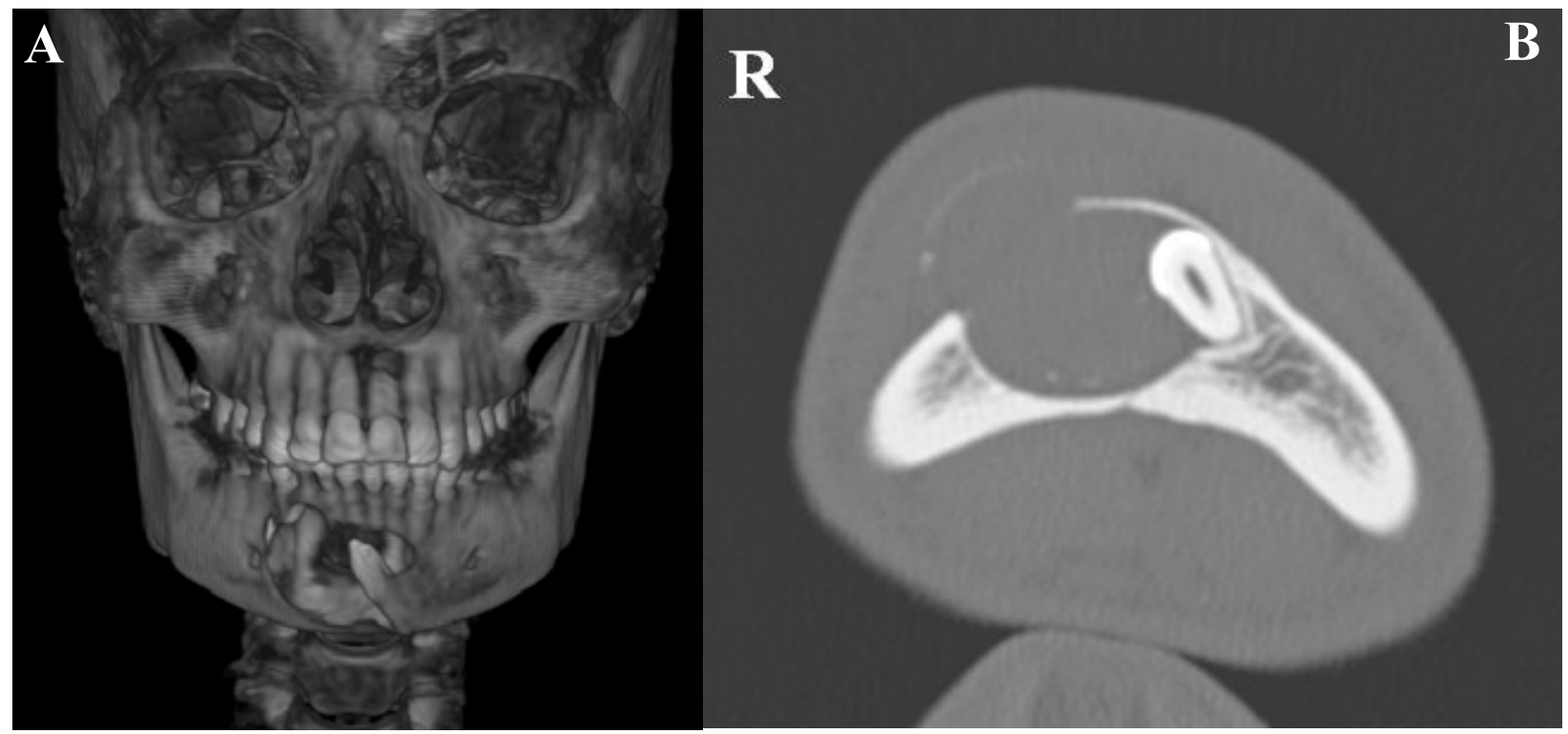

Fig. 2. A. 3-dimensional view showing the relation of the osteolytic lesion with the impacted canine

B. Axial CT scan revealing buccal cortical plate expansion and thinning of lingual cortical plates.

\section{Discussion}

AOT is defined as a tumor of odontogenic epithelium with duct-like structures and varying inductive changes in the connective tissue. (2) It was recognized as a dis- tinct clinicopathological entity by Stafne in 1948. (1) Although the original description of the lesion was given by Gosh in 1934 who refer to this lesion as an 'Adamantinoma of the upper jaw'. (1) Subsequently Bernier and

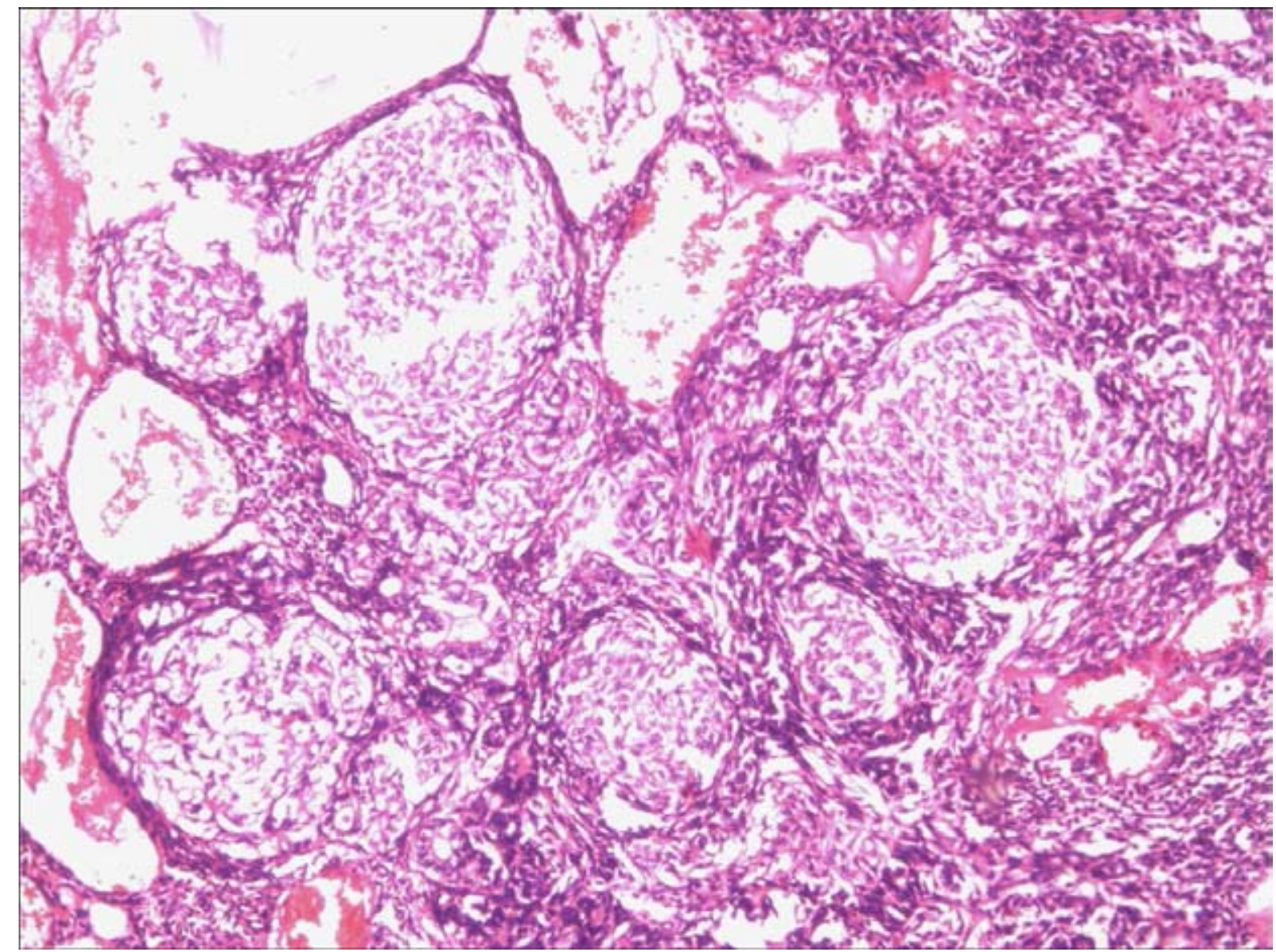

Fig. 3. Hematoxylin-eosin scanning (x100) showing clusters of cell rich epithelial nodules with droplets of eosinophilic material in between the spindle to polygonal cells. 
Tiecke in 1956 proposed the name 'Adenoameloblastoma' because of its adenoid configuration and resemblance to the ameloblastoma. (1) However, latter on it became apparent that the clinical and histological features of the AOT as well as its benign, hamartomatous biological behavior are distinctive and characteristic of its entity, and bears little resemblance to the ameloblastoma which is more aggressive neoplasm. For this reason, later workers advocated the usage of term 'Adenomatoid Odontogenic Tumor' in preference to Adenoameloblastoma so as to avoid misinterpretation and errors in treatment. (1) AOTs present a relative frequency of $2.2-7.1 \%$ among all odontogenic tumors. (5) It is more frequently seen in females and mostly occurs in the second decade of life. (1-5) The tumors are usually 1.5 to $3 \mathrm{cms}(2,4)$, but larger lesions have been have been reported in the literature. Swasdison et al. conducted a study analyzing 67 cases of AOT in Thai population and reported that the majority of cases presented with a painless swelling $(83.6 \%)$ and with duration of symptoms of 6 months and longer (57.14\%). (6) Progressive nasal obstruction is a common finding in lesions larger than $5 \mathrm{cms}$ located in maxilla. (5)

AOT can occur both intraosseously and extraosseously. Radiographically, intraosseous AOT can be divided into 2 types: follicular and extrafollicular. Intraosseous AOT are more frequent than the extraosseous variant with maximum cases showing the follicular variant. (6) Radiographically, the tumor usually appears unilocular, but cases with multilocular appearance have been reported. $(2,4)$ The tumor is commonly associated with an unerupted tooth simulating a dentigerous cyst. It may often appear complete radiolucent, however, they contain fine calcifications, a feature that may be helpful in differentiating AOT from dentigerous cyst. The radiolucency associated with an AOT may extend more apically than that of a dentigerous cyst. (1) Preoperatively, AOTs have been diagnosed as various types of disease, because of the variety of clinical and radiographic features of the lesions. $(2,8)$

The most frequent histological pattern is a proliferation of sheets, nests and cords of ameloblast-like cells supported by a scanty often hemorrhagic stroma. These cells may be organized to form whorls, rosettes, nodules or surround ovoid spaces to form duct-like structures. Additionally, "amyloid" like amorphous eosinophilic material may be found filling or lining the ductal lumens or disposed as intercellular droplets in cellular areas. (1, 8) Calcific material is often found throughout the tumor and appears to develop primarily as the junction between tumor epithelium and adjacent vascular stromal tissue. (1)

According to Giansanti et al. after local curettage of the tumor a number of cases were followed up for a period ranging from 1 to 10 years with no reported cases of recurrences. Indeed, Giansanti et al. reported that AOT was a completely benign tumor which never recurred once removed. $(4,7)$

Thus, a complete history with a systemic clinical diagnosis followed by appropriate radiographic investigation should help in diagnosing AOT and decrease the incidence of misdiagnosis for such cases and this in turn will help in preventing recurrences.

\section{References}

1. Siar $\mathrm{CH}, \mathrm{Ng} \mathrm{KH}$, Murugasu P. Adenomatoid odontogenic tumor: gross and histological examination of 45 cases. Singapore Med J.1987; 28: 180-9.

2. Sato D, Matsuzaka K, Yama M, Kakizawa T, Inoue T. Adenomatoid odontogenic tumor arising from the mandibular molar region: a case report and review of literature. Bull Tokyo dent Coll. 2004; 45: 223-7.

3. Jivan V, Altini M, Meer S, Mahomed F. Adenomatoid odontogenic tumor (AOT) originating in a unicystic ameloblastoma: a case report. Head and Neck Pathol. 2007; 1: 146-9.

4. Dayi E, Gürbüz G, Bilge OM, Ciftcioğlu MA. Adenomatoid odontogenic tumour (adenoameloblastoma). Case report and review of literature. Aust Dent J. 1997; 42: 315-8.

5. Vasconcelos BC, Frota R, Cardoso AB, Porto GG, Carneiro SC. Adenomatoid odontogenic tumor. Braz J Otorhinolaryngol. 2008; 74: 315 .

6. Swasdison S, Dhanuthai K, Jainkittivong A, Philipsen HP. Adenomatoid odontogenic tumors: an analysis of 67 cases in a Thai population. Oral Surg Oral Med Oral Pathol Oral Radiol Endod. 2008; 105: 210-5.

7. Giansanti JS, Someren A, Waldron CA. Odontogenic adenomatoid tumors (adenoameloblastoma). Survey of 3 cases. Oral Surg Oral Med Oral Pathol. 1970; 30: 69-88.

8. Vera-Sempere FJ, Artes-Martínez MJ, Vera-Sirera B, Bonet-Marco J. Follicular adenomatoid odontogenic tumor: Immunohistochemical study. Med Oral Patol Oral Cir Bucal. 2006; 11: e305-8. 